\title{
MicroRNA-30a regulates chondrogenic differentiation of human bone marrow-derived mesenchymal stem cells through targeting Sox9
}

\author{
HONGQI ZHANG, YUNJIA WANG, GUANTENG YANG, HONGGUI YU, \\ ZHENHAI ZHOU and MINGXING TANG \\ Department of Spine Surgery, Xiangya Hospital, Central South University, Changsha, Hunan 410008, P.R. China
}

Received September 30, 2018; Accepted July 5, 2019

DOI: $10.3892 /$ etm.2019.8148

\begin{abstract}
Cartilage injury is difficult to repair since the cartilage tissue lacks self-restoration ability. Improved formation of chondrocytes differentiated from the mesenchymal stem cells (MSC) by genetic regulation is a potentially promising therapeutic option. SOX9 is a critical transcription factor for mesenchymal condensation prior to chondrogenesis. Previous studies demonstrated that several microRNAs (miRNAs or miRs) play a critical role in the chondrogenic differentiation of MSCs. However, the interactional relations between miR-30a and SOX9 during chondrogenic differentiation of MSCs need to be further elucidated. In the present study, human bone marrow-derived mesenchymal stem cells have been isolated and induced into chondrogenic differentiation to imitate the cartilage formation in vitro. Additionally, the expression levels of several miRNAs that were reported to interact with the SOX9 3'untranslated region (UTR) were examined by using reverse transcription-quantitative PCR. The interactional relations between candidate miRNAs and SOX9 were verified with the transfection of a miRNA mimic or inhibitor and a luciferase reporter gene assay. The results indicate that miR-30a and miR-195 were consistently increased during MSC chondrogenic differentiation. Additionally, the binding of miR-30a to the SOX9 3'UTR was verified. Then, the authors upregulated the expression of miR-30a and found that MSC chondrogenic differentiation was inhibited. Taken together, the results of the present study demonstrate that miR-30a has a negative regulatory effect on MSC chondrogenic differentiation by targeting SOX9. Advances in epigenetic regulating methods will likely be the future of systemic treatment of cartilage injury.
\end{abstract}

Correspondence to: Dr Mingxing Tang, Department of Spine Surgery, Xiangya Hospital, Central South University, 87 Xiangya Road, Changsha, Hunan 410008, P.R. China

E-mail: philar1213@163.com

Key words: miR-30a, mesenchymal stem cells, SOX9, chondrogenic differentiation

\section{Introduction}

Cartilage injury is difficult to repair since the cartilage tissue lacks the self-restoration ability (1). Therefore, effective ways to repair a cartilage defect are a high priority research area (2). Despite some progress, current therapeutic methods are relatively inefficient and costly $(3,4)$. Improved formation of chondrocytes differentiated from the mesenchymal stem cells (MSCs) by genetic regulation is one of many potentially promising therapeutic options (5-7). Bone marrow-derived mesenchymal stem cells (BMSCs) are pluripotent stem cells that can differentiate into osteoblasts, chondrocytes, fibroblasts and adipocytes (8). Differentiation of MSCs into chondrocytes is a complex process strictly regulated by a complex transcriptional network (9). SOX9 is a critical transcription factor for mesenchymal condensation prior to chondrogenesis (10). A large body of evidence has suggested that the SOX9 expression is essential for the survival of chondrocytes in order to progress on to hypertrophy $(11,12)$.

MicroRNA (miRNA or miR) is a class of small non-coding RNA molecules (21-25 nt) (13). miRNAs play a critical role in many biological processes by promoting mRNA degradation by interacting with the 3'untranslated regions (UTRs) of specific mRNAs (14). Various miRNAs have been reported to differentially express during chondrogenic differentiation (15-17). Since miRNAs are small endogenous molecules, they can be used as a tool for genetic engineering $(18,19)$. miR-30a, miR-195, miR-216b and miR-15a were selected for the current study. miR-30a expression has been shown to increase in MSCs during chondrogenic differentiation and regulate chondrogenic differentiation (20). It was identified that miR-195 was downregulated in MSC during osteogenic differentiation and regulated MSC osteogenic differentiation (21). Moreover, it has been reported that miR-216b directly regulated SOX9 in cell proliferation (22). miR-15a expression decreased in adipose-derived MSC under normoxia treatment (23).

The aim of the current study was to elucidate the interaction between miR-30a and SOX9 during the chondrogenic differentiation of MSCs. Additionally, the authors investigated whether inhibition of miR-30a has an inhibitory effect on MSC chondrogenic differentiation via SOX9. 


\section{Materials and methods}

Ethics statement. The study protocol was approved by the Ethics Committee of Xiangya Hospital of Central South University (protocol ID: 201703358). Each bone marrow donor signed an informed consent before the study.

Cell culture. Human (h)BMSCs from healthy volunteers were extracted from bone marrow as described in our previous research (24). In brief, volunteers were recruited from patients undergoing bone marrow aspiration at Xiangya Hospital (Changsha, Hunan, China) from January 2015 to December 2016. Volunteers with immune system diseases and blood test abnormal were excluded. A total 8 patients ( 3 male and 5 female) from age 22-45 years old (mean age 36 years) were recruited. Bone marrow aspiration were performed to acquire the bone marrow. Ficoll density gradient media (density $1.077 \mathrm{~g} / \mathrm{cm}^{3}$; GE Healthcare) method was using to isolate hBMSC. Buffy coats, which including hBMSCs, were collected after centrifugation $\left(1,100 \mathrm{x} \mathrm{g} ; 20 \mathrm{~min}\right.$ at $\left.37^{\circ} \mathrm{C}\right)$. After isolation from the bone marrow aspirates, hBMSCs were incubated in a complete hBMSC medium supplemented with $10 \%$ fetal bovine serum (FBS; HUXMA-90011; Cyagen Biosciences, Inc.), 100 U/ml penicillin/streptomycin and glutamine at a density of $10^{7}$ cells per $100 \mathrm{~mm}$ dishes at $37^{\circ} \mathrm{C}$ in a $5 \% \mathrm{CO}_{2}$ incubator. The third generation of the cells was subjected to subsequent experiments. The HEK293 cell line (Thermo Fisher Scientific, Inc.) was cultured in high-glucose (4,500 mg/l) DMEM (HyClone; GE Healthcare Life Sciences) supplemented with $10 \%$ FBS and $1 \%$ penicillin/streptomycin at $37^{\circ} \mathrm{C}$ in a $5 \% \mathrm{CO}_{2}$ incubator.

Adipogenic, osteogenic and chondrogenic differentiation of MSCs. For adipogenic and osteogenic differentiation, the passage $3 \mathrm{hMSCs}$ were harvested with $0.25 \%$ trypsin and centrifuged $\left(168 \mathrm{x} \mathrm{g}\right.$ at $37^{\circ} \mathrm{C}$ for $4 \mathrm{~min}$, then suspended at $2 \times 10^{4}$ cells $/ \mathrm{cm}^{2}$ for subsequent differentiation. For adipogenic differentiation, hMSCs were cultured in an adipogenic differentiation medium (GUXMX-90031; Cyagen Biosciences, Inc.; kit components included mesenchymal stem cell adipogenic differentiation basal medium, 10\% FBS, $1 \%$ penicillin-streptomycin, $50 \mu \mathrm{g} / \mathrm{ml}$ glutamine, insulin $2 \mu \mathrm{l} / \mathrm{ml}$, isobutylmethylxanthine, dexamethasone $1 \mu \mathrm{l} / \mathrm{ml}$, dexamethasone $1 \mu \mathrm{l} / \mathrm{ml}$ and rosiglitazone $1 \mu \mathrm{l} / \mathrm{ml}$ ) at $37^{\circ} \mathrm{C}$ and $5 \% \mathrm{CO}_{2}$ incubator for 21 days.

Osteogenic differentiation was performed as described previously (24). Briefly, hBMSCs were incubated with Human Mesenchymal Stem Cell Growth Medium (HUXMX-90011; Cyagen Biosciences, Inc., kit components included human mesenchymal stem cell basal medium, $10 \%$ human mesenchymal stem cell-qualified fetal bovine serum, $1 \%$ penicillin-streptomycin and $2 \mathrm{mmol} / \mathrm{l}$ glutamine), after isolation from bone marrow at a $37^{\circ} \mathrm{C}$ and $5 \% \mathrm{CO}_{2}$ incubator.

For chondrogenic differentiation, $1 \times 10^{6} \mathrm{hMSCs}$ were suspended and centrifuged $\left(300 \mathrm{x} \mathrm{g} ; 4 \mathrm{~min} ; 37^{\circ} \mathrm{C}\right)$ to form a cell mass in a $\mathrm{V}$-shape polypropylene culture tube in a $37^{\circ} \mathrm{C}$ incubator in $5 \% \mathrm{CO}_{2}$. The cell mass was incubated in a Mesenchymal Stem Cell Chondrogenic Differentiation Medium (GUXMX-90041, Cyagen Biosciences, Inc.; kit components included $194 \mathrm{ml}$ basal medium, $100 \mathrm{nM}$ dexamethasone, $50 \mu \mathrm{g} / \mathrm{ml}$ ascorbate, $6.25 \mu \mathrm{g} / \mathrm{ml}$ insulin-transferred
selenium-A, $100 \mu \mathrm{g} / \mathrm{ml}$ sodium pyruvate, $50 \mu \mathrm{g} / \mathrm{ml}$ proline and $10 \mathrm{ng} / \mathrm{ml}$ TGF- $\beta 3$ ) in a V-shape polypropylene culture tube for 21 days. The induction medium was changed every 2 days.

Staining. Oil red O staining, alizarin red S staining and toluidine blue staining were performed to assess the multi-differentiation potential of MSCs. For Oil red O staining, cell at $80 \%$ confluence were fixed with $4 \%$ paraformaldehyde solution for $15 \mathrm{~min}$ at room temperature and incubated in $0.18 \%$ Oil red O (Sigma-Aldrich; Merck KGaA) solution for $30 \mathrm{~min}$ at room temperature. For alizarin red $\mathrm{S}$ staining, cells were fixed in $4 \%$ paraformaldehyde solution for $15 \mathrm{~min}$ and incubated in 3\% alizarin red S (Sigma-Aldrich; Merck KGaA) solution for $30 \mathrm{~min}$ at room temperature. For toluidine blue staining, the cell mass was fixed in 4\% paraformaldehyde solution for $24 \mathrm{~h}$, dehydrated by in an ethanol gradient (70-96\%) at room temperature then paraffin embedded. Sections $(10 \mu \mathrm{m})$ were stained with $0.5 \%$ toluidine blue (Sigma-Aldrich; Merck $\mathrm{KGaA}$ ) solution for $20 \mathrm{~min}$ at room temperature. The stained cells were observed under a phase contrast microscope (Olympus Corporation).

Flow cytometry analysis. Flow cytometry analysis was performed using an hMSC characterization kit (HUXMX-09011; Cyagen Biosciences, Inc.) to detect the MSC surface-specific antigens. In brief, MSCs were suspended $\left(3 \times 10^{6}\right.$ cells $\left./ \mathrm{ml}\right)$ and diluted with PBS with $0.1 \%$ BSA, mixed $100 \mu 1$ cell suspension with $2 \mu \mathrm{l}$ various fluorescently labeled monoclonal antibodies, including anti-human CD45, CD14, CD44, CD105, CD90 and CD34, and then incubated at room temperature for $30 \mathrm{~min}$ in the dark. The cells were washed by PBS with $0.1 \%$ BSA twice and then centrifuged $(250 \mathrm{x}$ g; $5 \mathrm{~min}$; room temperature). The supernatant was discarded and cells re-suspended with PBS with $0.1 \%$ BSA and incubated with $2 \mu \mathrm{l}$ fluorescein isothiocyanate (FITC)/propidium iodide (PI) goat anti-mouse IgG antibodies at room temperature for $30 \mathrm{~min}$ in the dark. Then samples were washed with $1 \mathrm{ml}$ PBS with $0.1 \%$ BSA twice, centrifuged $\left(250 \mathrm{x} \mathrm{g} ; 5 \mathrm{~min} ; 4^{\circ} \mathrm{C}\right)$. The supernatant was discarded and cells were resuspended with $400 \mu 1$ PBS with $0.1 \%$ BSA and analyzed immediately using a BD FACSCalibur flow cytometer (BD Biosciences). An unstained sample $\left(1 \times 10^{6}\right)$ was used as a negative control; the data were analyzed using BD FACSuite software v1.0 (BD Biosciences).

Cell transfection. Prior to chondrogenic differentiation, MSCs were transfected with the miR-30a mimic, miR-30a inhibitor or their negative controls by using Lipofectamine 2000 (Invitrogen; Thermo Fisher Scientific, Inc.) according to the manufacturer's protocol. The miR-30a mimic, miR-30a inhibitor and their negative controls were purchased from GenePharma Co., Ltd. and the sequences were as follows: miR-30a mimic, 5'-UGUAAACAUCCUCGACUGGAAG-3' (sense); miR-30a inhibitor, 5'-CUUCCAGUCGAGGAUGUU UACA-3'; negative control, 5'-UUCUCCGAACGUGUCACG UTT-3' (sense). In brief, MSCs were seeded into six-well plate $\left(1 \times 10^{5} /\right.$ well) and were subjected to transfection after $24 \mathrm{~h}$. Transfection of $50 \mathrm{nM}$ miR-30a mimic, miR-30a inhibitor and their negative controls were performed. The cells were incubated at a $37^{\circ} \mathrm{C}$ and $5 \% \mathrm{CO}_{2}$ incubator for $4 \mathrm{~h}$. In order 
to exclude the potential influence of transfection reagent, a MOCK transfection group which transfected without RNA.

The transfection efficiency was evaluated by the visualization of fluorescence by Cy3-labeled miRNA transfection. In brief, Cy3-labeled miRNA (cat. no. AM17120; Thermo Fisher Scientific, Inc.) was designed for monitoring transfection efficiency before miRNA mimic or inhibitor transfection experiments. MSCs were seeded into six-well plates $\left(1 \times 10^{5} /\right.$ well) and were subjected to transfection after $24 \mathrm{~h}$. A total of $50 \mathrm{nM} \mathrm{Cy3-labeled} \mathrm{miRNA}$ was transfected with Lipofectamine 2000 at a $37^{\circ} \mathrm{C}$ and $5 \% \mathrm{CO}_{2}$ incubator. After $4 \mathrm{~h}$ of transfection, cells with fluorescence were observed and counted under a laser-scanning microscope with x20 magnification (FV10-ASW 1.7; Olympus Corporation). The transfection efficiency was $50 \%$ (result not shown). A total of $24 \mathrm{~h}$ after the transfection, the cells were subjected to the chondrogenic differentiation assay with subsequent detection of the results.

Luciferase reporter assay. Pairing between miR-30a and SOX9 mRNA was reported by the miRNA databases, miRBase (http://www.mirbase.org/) and TargetScan (http://www. targetscan.org/vert_72/). SOX9 3'UTR and 5-bp base mutated SOX9 3'UTR regions were amplified and cloned into the pGL4.26 luciferase reporter plasmid (Promega Corporation). HEK293 cells were divided into 6 groups randomly and co-transfected with a random DNA sequence (5'-UUCUCC GAACGUGUCACGUTT-3'), the miR-30a mimic or the negative control mimic and the wild-type or mutant recombinant reporter plasmid at $40 \%$ confluence using Lipofectamine 2000. Luciferase reporter assays were performed $24 \mathrm{~h}$ post-transfection using Dual Luciferase ${ }^{\circledR}$ Reporter assay system (Promega Corporation) according to the manufacturer's protocol. Luciferase activities were determined and the ratio of firefly luciferase to Renilla luciferase activity was calculated. All transfection experiments were performed in triplicate.

Western blotting. MSCs were harvested in a lysis buffer (Beyotime Institute of Biotechnology) containing protease inhibitors and then centrifuged at $10,000 \mathrm{x}$ g for $20 \mathrm{~min}$ at $4^{\circ} \mathrm{C}$. Protein concentration was determined with the BCA Protein Assay reagent (Thermo Fisher Scientific, Inc.) according to the manufacturer's protocol. After quantification, $20 \mu \mathrm{g}$ of the sample was loaded into $10 \%$ gels, separated using SDS-PAGE and then transferred to a PVDF membrane (Bio-Rad Laboratories, Inc.). After blocking with 5\% non-fat milk in Tris-buffered saline and Polysorbate 20 for $1 \mathrm{~h}$ at room temperature, the blots were incubated with the primary antibodies (all Abcam) including SOX9 (1:1,000; cat. no. ab185966), aggrecan (1:1,000; cat. no. ab3778), collagen II $(1: 1,000$; cat. no. ab34712), $\beta$-catenin (1:500; cat. no. ab32572) and $\beta$-actin (1:5,000; cat. no. ab8226). After incubating the membrane with the appropriate secondary antibody $(1: 10,000$; goat anti-rabbit IgG antibody, cat no. ab150077 and goat anti-mouse IgG antibody, cat no. ab150117; Abcam) in the blocking buffer for $1 \mathrm{~h}$ at room temperature, protein expression was quantified by Chemiluminescence Protein Detection Module (Millipore, Inc.). $\beta$-actin were used as an internal reference. Band intensity was determined by Image J (v 1.4.0; National Institutes of Health) program.
Reverse transcription-quantitative PCR (RT-qPCR). Total RNA was extracted from the MSCs using TRIzol reagent (Invitrogen; Thermo Fisher Scientific, Inc.) according to the manufacturer's protocol. For reverse transcription of miRNA, miRNA cDNA Synthesis Kit (Thermo Fisher Scientific, Inc.) was used. In brief, mature miRNAs were extended by an adaptor sequence of $3^{\prime}$ poly-A tailing and $5^{\prime}$ ligation prior to reverse transcription. Then a standard reverse transcription reaction $\left(42^{\circ} \mathrm{C}\right.$ for $15 \mathrm{~min}, 85^{\circ} \mathrm{C}$ for $5 \mathrm{~min}$, then hold at $4^{\circ} \mathrm{C}$ ) was performed. qPCR was performed using a SYBR Green Realtime PCR master mix (Takara Bio, Inc.) in PTC-220 Real-Time PCR Machine (Bio-Rad Laboratories, Inc.) according to the manufacturer's protocol. U6 was used as an internal reference for miRNA expression analysis. The thermocycling conditions were as follows: Incubation at $95^{\circ} \mathrm{C}$ for $10 \mathrm{~min}$, followed by 40 cycles of $95^{\circ} \mathrm{C}$ for $10 \mathrm{sec}, 60^{\circ} \mathrm{C}$ for $20 \mathrm{sec}$ and $72^{\circ} \mathrm{C}$ for $30 \mathrm{sec}$, then incubation at $4^{\circ} \mathrm{C}$ for $5 \mathrm{~min}$. Specific primers were used for quantitative PCR as follows: U6 forward, 5'-GCTTCGGCAGCACATATACTAAAAT-3' and reverse, 5'-CGCTTCACGAATTTGCGTGTCAT-3'; miR-30a forward, 5'-ACACTCCAGCTGGGTGTAAACATCCTCGAC TG-3' and reverse, 5'-CTCAACTGGTGTCGTGGA-3'; miR-195 forward, 5'-GTCGTATCCAGTGCGTGTCGTGGAGTCGGC AATTGCACTGGATACGAGCCAAT-3' and reverse, 5'-GGG GTAGCAGCAGCACAGAAAT-3'; miR-216b forward, 5'-GCC GCGCTAAAGTGCTTATAGTG-3' and reverse, 5'-CACCAG GGTCCGAGGT-3'; and miR-15a forward, 5'-TTGTAATAC GACTCACTATAGGGAGAGAGTCGATGTGTTCTTC-3' and reverse 5'-ACATGGGTTTAGCCATCCAGAAACCCACC-3'. The relative expression levels of the miRNAs were calculated using the $2^{-\triangle \Delta \mathrm{Cq}}$ method (25).

Cell proliferation. To assess cell proliferation after the miR-30a mimic transfection, Cell Counting Kit-8 (CCK-8; Dojindo Molecular Technologies, Inc.) assay was performed according to the manufacturer's protocol. Briefly, MSCs $\left(5 \times 10^{3}\right.$ cells/well) were transfected with the $5 \mathrm{nM}$ miR-30a mimics and negative controls and plated in 96-well plates for 24 or $48 \mathrm{~h}$ at $37^{\circ} \mathrm{C}$. Then, $10 \mu \mathrm{l}$ of the CCK-8 reagent (Dojindo Molecular Technologies, Inc.) was added to each plate for $4 \mathrm{~h}$ at $37^{\circ} \mathrm{C}$. Absorbance at $450 \mathrm{~nm}$ was determined and used to calculate cell viability.

Immunofluorescence analysis. Immunofluorescence analysis was performed to determine SOX9 translocation in transfected MSCs. After 10 days of chondrogenic differentiation, MSCs were fixed with $4 \%$ paraformaldehyde $(\mathrm{pH} \mathrm{7.4)}$ for $10 \mathrm{~min}$ at room temperature and permeabilized with $0.1 \%$ Triton $\mathrm{X}-100$ for $5 \mathrm{~min}$. Then, the slides were incubated with SOX9 primary antibodies (1:200) for $1 \mathrm{~h}$ at room temperature. Slides were washed and incubated with a fluorescently labeled secondary antibody (1:200, goat anti-rabbit IgG secondary antibody, cat. no. A32731, Invitrogen; Thermo Fisher Scientific, Inc.) for $1 \mathrm{~h}$ at room temperature in the dark, and the nuclei were stained with DAPI (Sigma-Aldrich; Merck KGaA) for $30 \mathrm{~min}$ at room temperature. The images were acquired using a laser-scanning microscope (FV10-ASW 1.7; Olympus Corporation).

Statistical analysis. The results are presented as mean \pm SD and analyzed using SPSS 13.0 software (SPSS, Inc.). Changes between the groups were assessed using analysis of variance 


\section{A}

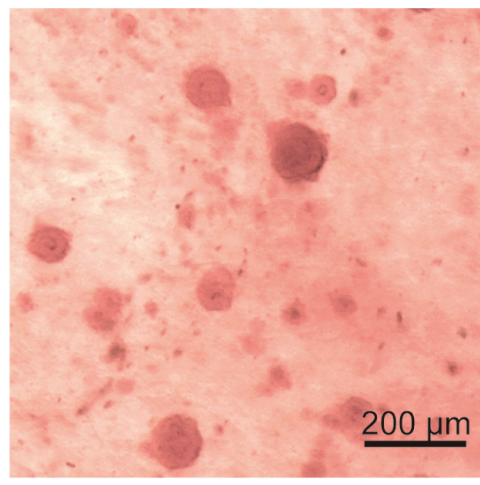

Alizarin red
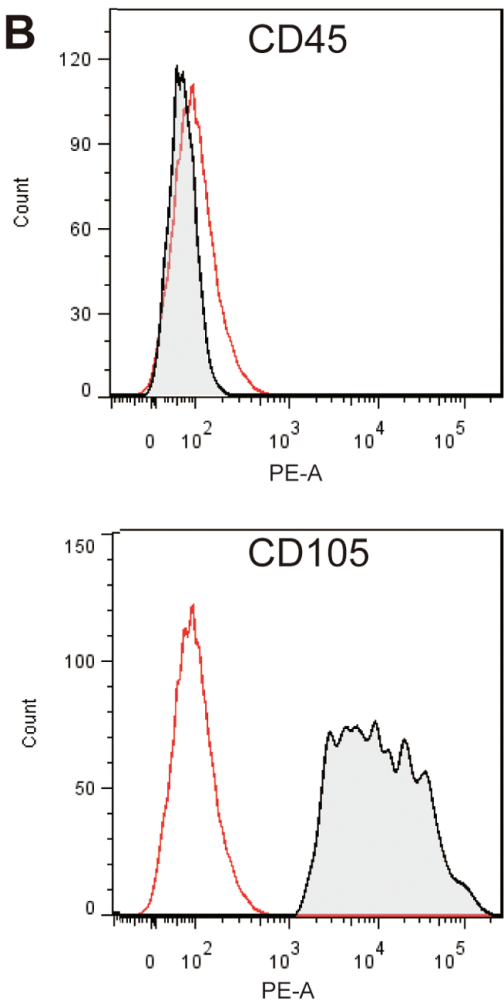

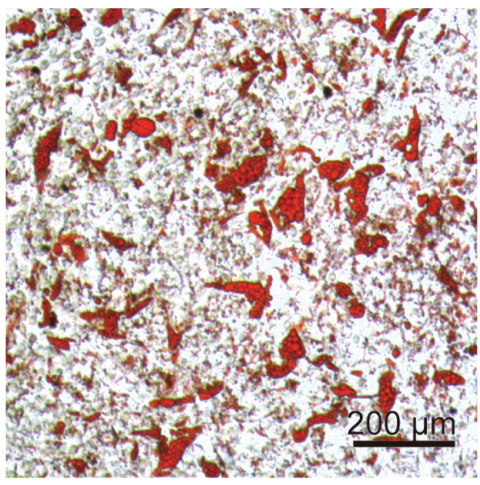

Oil red O
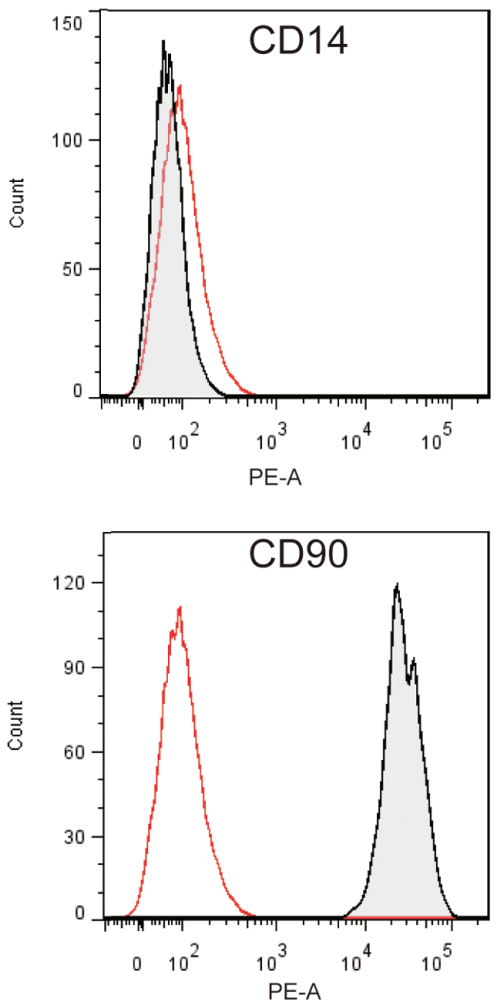

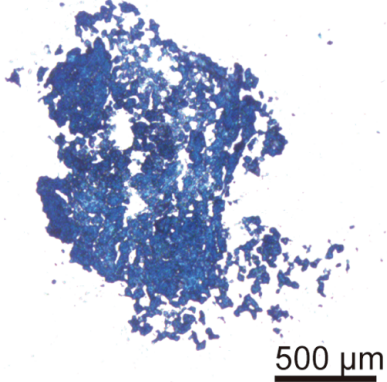

Toluidin blue
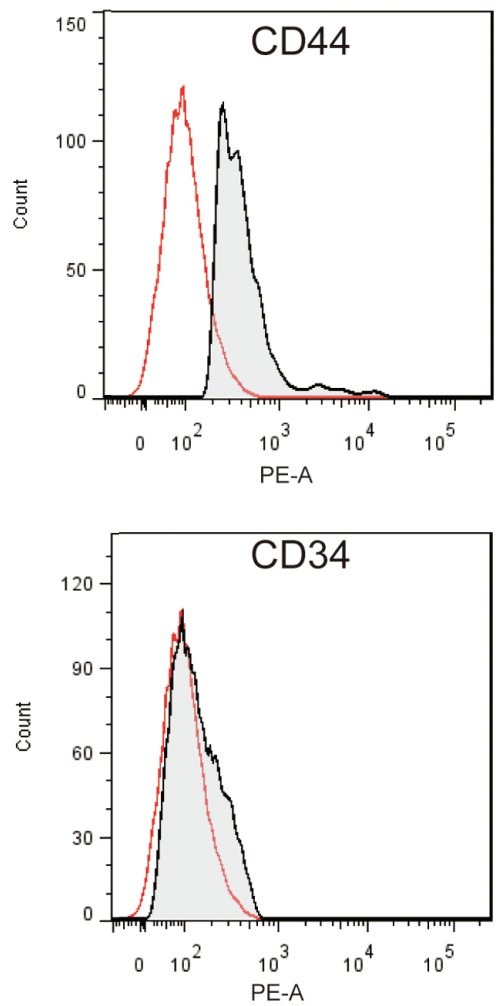

Figure 1. Characterization of hBMSCs. (A) MSCs from the human bone marrow were isolated and cultured. Alizarin red staining, Oil red O staining and toluidine blue staining were performed to assess the osteogenic, adipogenic and chondrogenic differentiation potential, respectively. (B) The cell membrane markers of MSCs were determined by flow cytometry. Surface markers CD45, CD14, CD44, CD105, CD29 and CD34 were detected and analyzed (n=3). MSCs, mesenchymal stem cells; hBMSCs, bone marrow-derived mesenchymal stem cells; CD, cluster of differentiation.

followed by Bonferroni's post-hoc test. A value of $\mathrm{P}<0.05$ was considered to indicate a statistically significant difference.

\section{Results}

Characterization of cultured MSCs. To identify the cultured MSCs extracted from the bone marrow, multi-lineage differentiation capacity and expression of the characteristic cell surface MSC markers were determined by flow cytometric analysis after the induction of various types of differentiation. As shown in Fig. 1, osteogenic, adipogenic and chondrogenic differentiation were induced in the cultured MSCs. After 21 days of differentiation, the results of alizarin red, oil red $\mathrm{O}$ or toluidine blue staining were positive and revealed that cultured MSCs have the multi-lineage differentiation ability (Fig. 1A). CD90, CD105 and CD44 are specific cell surface markers of MSCs while CD34, CD14 and CD45 are the hematopoietic stem cell markers that were not expressed in the MSCs (26). Flow cytometry analysis showed that the cultured MSCs expressed CD90, CD105 and CD44, but did not express CD34, CD14 and CD45 (Fig. 1B).

Expression of SOX9 and candidate miRNAs during MSC chondrogenic differentiation. SOX9 plays the critical role in MSC chondrogenic differentiation (27-29); therefore, the authors decided to determine the expression levels of SOX9 at various stages of MSC chondrogenic differentiation by western blotting. During chondrogenesis, SOX9 expression was initially 
A

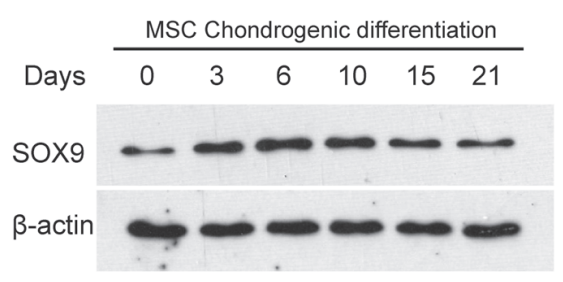

C

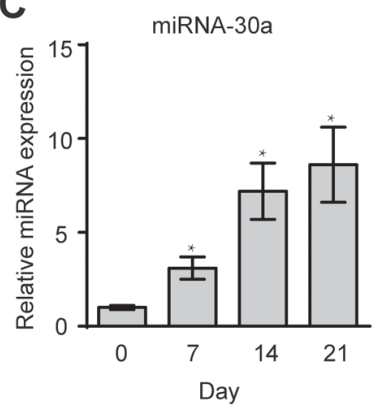

D

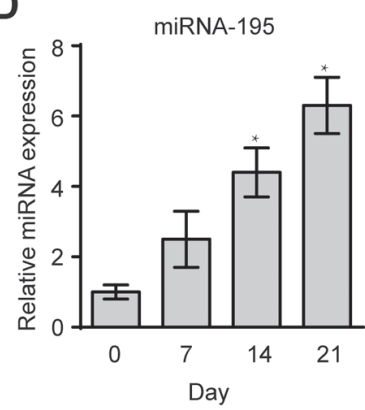

B

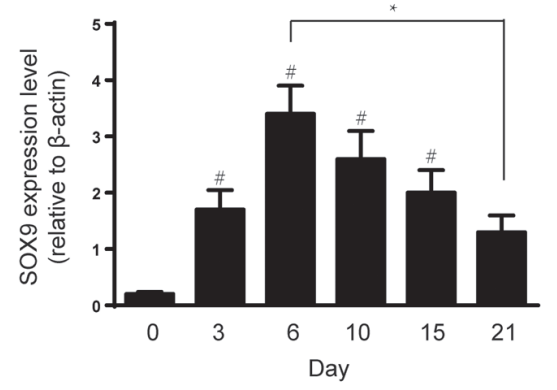

E

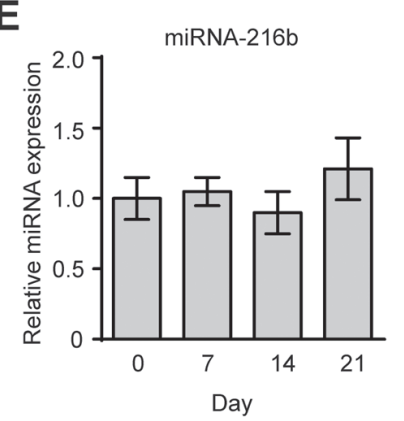

$\mathbf{F}$

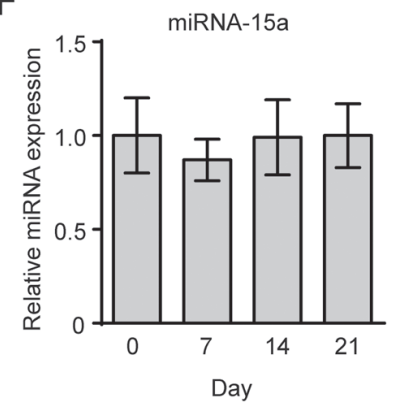

Figure 2. Differential expression of Sox9 and SOX9-associated miRNAs during chondrogenic differentiation of MSCs. (A) Western blotting and (B) quantification of protein expression. $\beta$-actin was used as an internal reference. One-way ANOVA, ${ }^{*} \mathrm{P}<0.05$ between two indicated groups; ${ }^{*} \mathrm{P}<0.05$ vs. day 0 group. (C-F) After induction of chondrogenic differentiation (7-21 days), the levels (C) miR-30a, (D) miR-195, (E) miR-216b and (F) miR-15a were determined by reverse transcription-quantitative PCR. Data are presented as mean \pm SD of three independent experiments. One-way ANOVA, ${ }^{\text {P }}<0.05$ vs. day 0 . SOX9, transcription factor SOX-9; MSCs, mesenchymal stem cells; miR or miRNA, microRNA.

increased and then was decreased when compared to the initial level (Fig. 1A and B). The goal of the present study was to identify miRNAs that specifically interact with the SOX9 gene during MSC chondrogenic differentiation. Hence, several candidate SOX9-associated miRNAs were chosen from the databases and the literature including miR-30a, miR-195, miR-216b and miR-15a $(20,22,23,30,31)$. They were selected from several studies about altered miRNA expression during chondrogenic differentiation, all the candidate miRNAs were predicted to interact with SOX 9 by the aforementioned miRNA databases. The expression levels of the candidate miRNAs were quantified by RT-qPCR. The expression levels of miR-30a and miR-195 consistently increased during MSC chondrogenic differentiation compared with the untreated group (Fig. 2C-D). In addition, the expression levels of miR-216b and miR-15a were not changed during chondrogenic differentiation (Fig. 2E-F).

miR-30a inhibits SOX9 expression in a cell line and in MSCs. The data indicate that SOX 9 mRNA possesses a potential miR-30a binding site in the 3'UTR (Fig. 3A). To confirm this prediction, a luciferase reporter gene assay was performed using the luciferase reporter vectors containing the wild-type SOX9 3'UTR and the 5-bp base mutated SOX9 3'UTR. The results indicate that the fluorescence of the wild-type SOX9 3'UTR reporter plasmid was significantly decreased after the miR-30a mimic transfection, while luciferase activity was not affected in the case of the mutant reporter plasmid (Fig. 3B). To further validate this prediction, the SOX 9 expression levels were determined by western blotting in transfected MSCs. However, miR-30a transfection efficiency was determined before detection of interaction relationship between miR-30a and SOX9. After miR-30a mimic transfection, the increased expression of miR-30a was determined by RT-qPCR (Fig. 3C). The expression of SOX9 was significantly decreased in miR-30a mimic-transfected MSCs compared with the control group (Fig. 3D and E). The results confirmed that miR-30a can bind to the SOX9 3'UTR.

Overexpression of miR-30a suppresses chondrogenesis. Since miR-30a was upregulated in the early stage of MSC chondrogenic differentiation, the authors speculated that miR-30a may affect MSC chondrogenic differentiation via SOX9. To verify this hypothesis, MSCs were transfected with the miR-30a mimics, miR-30a inhibitors or negative controls. First, the effect of the miR-30a mimic transfection on the cell viability was determined. The results indicate that miR-30a overexpression had no cytotoxic effect in the MSCs (Fig. 4A). Subsequently, the miR-30a mimics were transfected into the MSCs on day 7 of chondrogenic differentiation. Toluidine blue staining showed a marked decrease in the synthesis of the extracellular matrix on day 21 of the chondrogenic differentiation (Fig. 4B). Additionally, the translocation of SOX9 was detected by immunofluorescence in the MSCs transfected with the miR-30a mimic or miR-30a inhibitor on day 7 of chondrogenic differentiation. The nuclear translocation of SOX9 could be inhibited by the miR-30a mimic transfection. On the contrary, the miR-30a inhibitor transfection increased the translocation of SOX9 into the nucleus (Fig. 4C). Furthermore, the levels of the cartilage marker proteins including aggrecan and collagen II (32) were evaluated by western blotting after the transfections. Western blotting results demonstrated that cartilage markers were enhanced in the miR-30a inhibitor group compared with the miR-30a mimic group (Fig. 4D). 
A

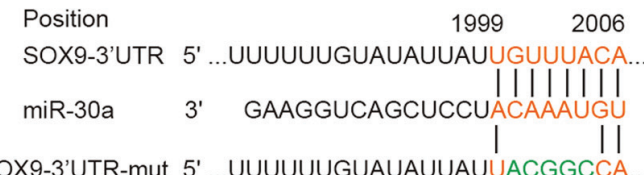

B

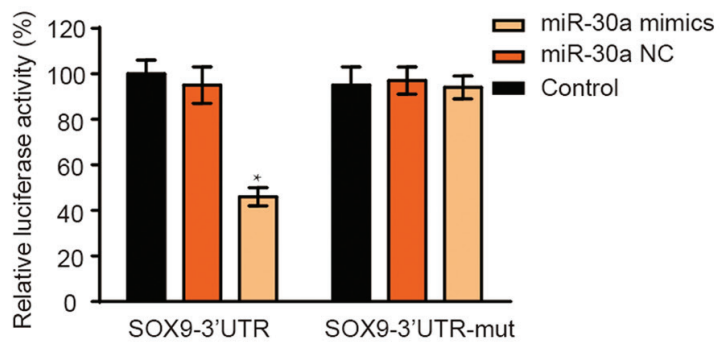

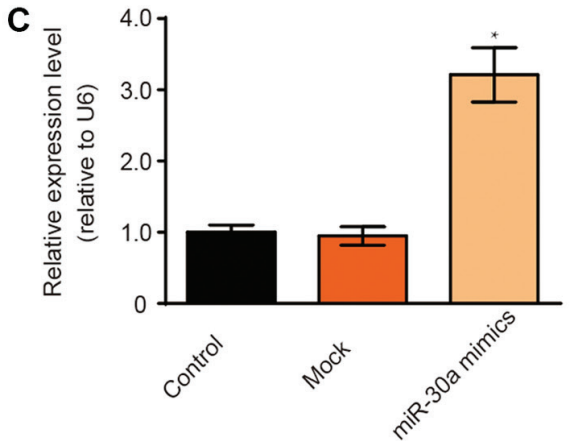

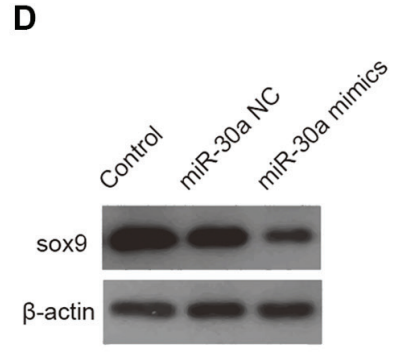

$E$

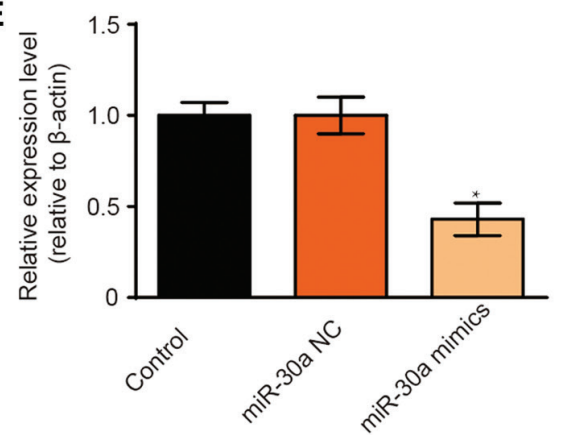

Figure 3. Effect of miR-30a on SOX9 expression. (A) Alignment of sequences of the wild-type SOX9, miR-30a and mutant SOX9 3'UTR. The lines between the sequences shows how complementary the sequences are. The mutant SOX9 3'UTR contains 5 nucleotide mutations, which are indicated in green. (B) Relative luciferase activity was assayed after the 293 cells were co-transfected with a random DNA sequence or the miR-30a mimic or the negative control mimic and the reporter plasmid with the wild-type or mutant SOX9 3'UTR. Data are presented as mean \pm SD of three independent experiments. One-way ANOVA, ${ }^{*} \mathrm{P}<0.05$ versus control. (C) MSCs were transfected with either miR-30a mimic or transfection reagents, the levels of miR-30a were determined by reverse transcription-quantitative PCR. (D) Western blotting and (E) quantification of protein expression. $\beta$-actin was used as an internal reference. Data are presented as mean \pm SD of three independent experiments. One-way ANOVA, ${ }^{*} \mathrm{P}<0.05$ vs. control. SOX 9 , transcription factor SOX-9; MSCs, mesenchymal stem cells; miR or miRNA, microRNA; NC, negative control; UTR, untranslated region.

Finally, the protein levels of $\beta$-catenin, the key factor of the Wnt signaling pathway (33), were examined by western blotting to verify the critical role of SOX9 in the effect of miR-30a on chondrogenesis. The result showed that $\beta$-catenin was inhibited by miR-30a overexpression (Fig. 4D).

\section{Discussion}

Molecular mechanism underlying MSC chondrogenesis remains poorly understood (34); thus, the application of mesenchymal cells for cartilage regeneration and repair is still not feasible (35). In the present study, hBMSCs have been isolated and induced into chondrogenic differentiation to imitate the cartilage formation in vitro. The authors of the current study determined typical MSC phenotype by the expression of characteristic cell surface markers, including CD45, CD14, CD44,CD105,CD90 and CD34, and multi-lineage differentiation capacity. CD34 is expressed in hematopoietic stem cell(36).CD45 and CD14 are lineage commitment cell antigens (37). In addition, CD44, CD105 and CD90 were important surface markers express in MSC (38). Moreover, the SOX9 transcription factor is essential for the mesenchymal cell aggregation prior to chondrogenesis (39). Hence, the expression levels of several miRNAs that were reported to interact with the SOX9 3'UTR were examined. The results indicate that miR-30a and miR-195 were consistently increased during MSC chondrogenic differentiation. Additionally, the binding of miR-30a to the SOX9 3'UTR region was verified. Then, the expression of miR-30a was upregulated and it was found that MSC chondrogenic differentiation was inhibited. The results of the current study demonstrate that miR-30a can inhibit MSC chondrogenic differentiation via SOX9.
Chondrogenic differentiation of the MSCs is an intricate process that is regulated by multiple factors $(40,41)$. In this process, SOX9 is the master regulator since high levels of SOX9 play an essential role in the aggregation of MSCs prior to cartilage formation. SOX9 expression was initially increased and then decreased during chondrogenesis. SOX9 activated the expression of numerous chondrocyte-specific genes including collagen (Col)2a1, Col9a1, Col11a2 and aggrecan, and the accumulation of the extracellular matrix (42). This importance of SOX9 in chondrogenesis suggests that SOX9 can be used as a regulatory factor. Therefore, the current study focused on SOX9 to identify its epigenetic regulatory factors.

miRNAs play an important role in stem cell self-renewal and differentiation as endogenous post-transcriptional regulators (43). Recently, several studies suggested that miRNAs may be involved in MSC chondrogenic differentiation (44-46). During the chondrogenic differentiation of MSCs, various miRNAs display differential expression patterns and can regulate the differentiation process by inhibiting the expression of specific target genes. For example, miR-140, miR-483, miR-495 and miR-410 regulated chondrogenic differentiation by modulating HDAC4 (47), SMAD4 (16), SOX9 (17) and Wnt3a (48), respectively. Based on a review of the published literature and of miRNA databases, the authors of the current study selected several miRNAs that were reported to regulate SOX9 and are expressed in various types of stem cells $(21,23)$. miR-30a, miR-195, miR-216b and miR-15a were selected for this study. The SOX9 expression pattern during the chondrogenic differentiation was similar to a parabola (49). In the current study, the expression levels of SOX9 decreased in the later stages of the chondrogenic differentiation while the levels 


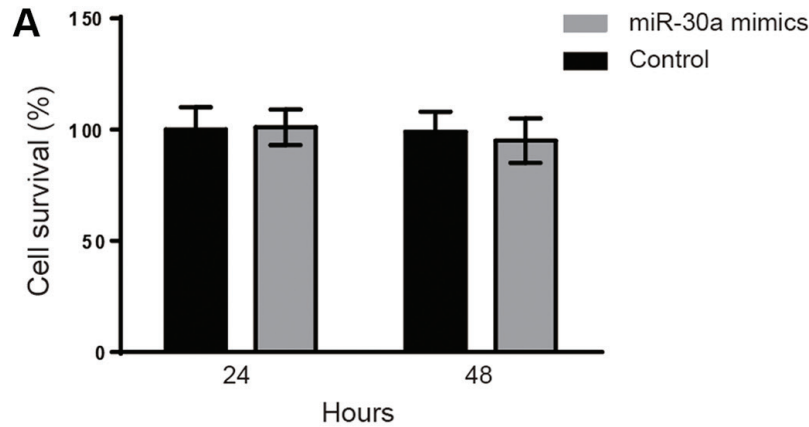

C

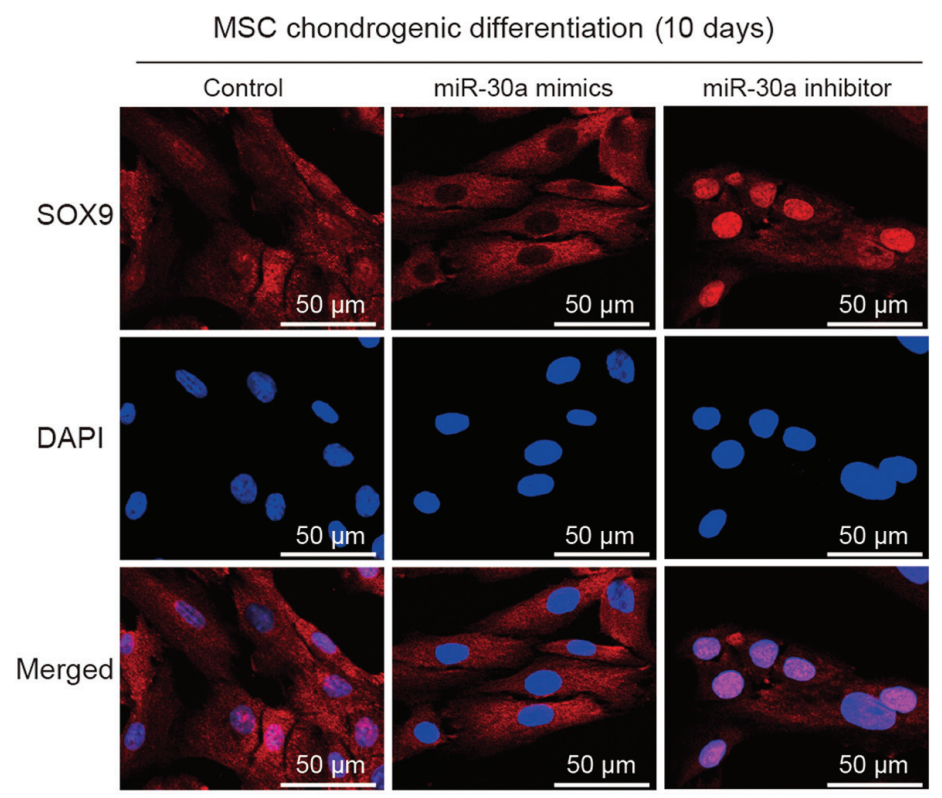

B MSC chondrogenic differentiation (21 days)

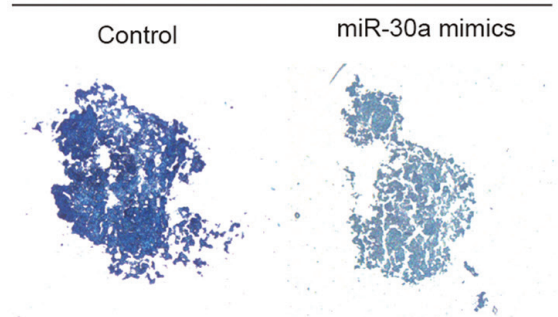

D

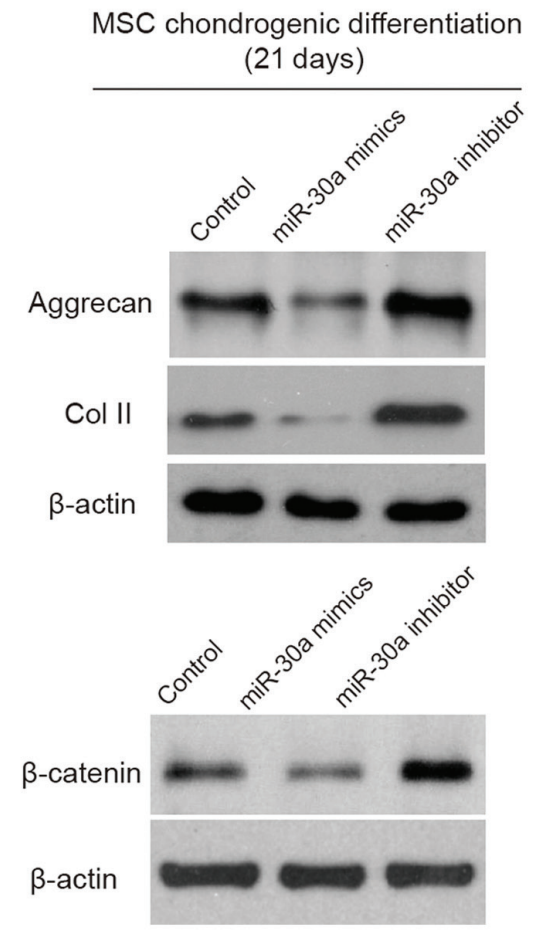

Figure 4. Effect of miR-30a on MSC chondrogenic differentiation. (A) MSCs were transfected with the miR-30a mimics for 24 or 48 h and cell viability was determined by a Cell Counting Kit- 8 . The mean value of the control group was assumed as $100 \%$. Data are presented as mean \pm SD of three independent experiments. One-way ANOVA. (B) MSCs were transfected with the miR-30a mimics. The extracellular matrix was detected by toluidine blue staining. (C) SOX9 translocation in transfected MSCs was detected by immunofluorescence. SOX9 staining corresponds to red fluorescence. Nuclei were stained with DAPI and are shown as blue fluorescence. Scale bars $=50 \mu \mathrm{m}$. (D) Western blotting detection of the cartilage markers and Wnt signaling factors including aggrecan, collagen II and $\beta$-catenin. $\beta$-actin was used as an internal reference.

of miR-30a and miR-195 gradually increase, which is consistent with a previous study that showed that increased miR-30a and decreased SOX9 were detected in primary chondrocytes from cartilage taken from patients with osteoarthritis (20). In the present study, miR-30a upregulation was identified and it was shown to suppress SOX9 expression. A luciferase assay also demonstrated that miR-30a specifically targeted the 3'UTR of SOX9.

Hence, it was hypothesized that these miRNAs inhibit sustained expression of SOX9 to regulate chondrogenesis. However, it remained unclear whether they can regulate MSC chondrogenic differentiation and what are the specific mechanisms of the process. Therefore, a series of experiments were carried out and the results indicated that overexpression of miR-30a reduced the synthesis of extracellular matrix components, indicating that miR-30a can inhibit MSC chondrogenic differentiation. In the process, miR-30a inhibited SOX9 protein expression by directly binding to the SOX9 mRNA
3'UTR region. The authors speculated that the miR-30a level gradually increases during MSC chondrogenic differentiation and subsequently inhibits the sustained expression of SOX9 leading to a decrease in the Sox 9 expression during the subsequent stage of differentiation. SOX9 translocation from cytoplasmic to nucleus was detected by immunofluorescence after miR-30a inhibitor transfection. As SOX9 was a transcription factor it binds to the promoter of specifically genes in the nucleus (50). The SOX9 nuclear translocation means the enhancement of the SOX9 function. In addition, miR-30a has been reported to serve as a prognostic factor in urothelial carcinoma of bladder and left ventricular dysfunction after acute myocardial infarction $(51,52)$. As the Wnt signaling pathway serves a significantly important role in cartilage developments and $\beta$-catenin is a part of the pathway (53), its' expression was assessed. $\beta$-catenin expression was decreased after miR-30a mimic transfection, which means the activation of Wnt signaling pathway is influenced by miR-30a levels. 
$\beta$-catenin accumulation in the cytoplasm and nuclear translocation of $\beta$-catenin are key procedures in the activation of canonical Wnt pathway (53).

The present study had some limitations. Firstly, the results obtained in the current study are not likely to imitate the effects in vivo, although MSCs were employed. Thus the results need to be validated with animal studies. Secondly, miR-30a was selected from a limited candidate group and other potential miRNAs may exist, which need to be explored in more studies. Thirdly, it is more convincing to add the control images of various MSC differentiation stages to elucidate the SOX9 expression during MSC differentiation, which is lacking in the present study. Lastly, the cytoplasmic and nuclear SOX9 levels were not verified by western blotting.

In conclusion, the current study showed that miR-30a has a negative regulatory effect on MSC chondrogenic differentiation by targeting SOX9. Interfering with endogenous expression of miR-30a and attenuating its inhibitory effect on SOX9 may be possible. Therefore, miR-30a and its mechanism of action may provide for a novel strategy for the treatment of cartilage-related diseases.

\section{Acknowledgements}

Not applicable.

\section{Funding}

This work was supported by the Chinese National Natural Science Foundation (grant nos. 81472145 and 81772298) and the Central South University Graduate Innovation Fund (grant no. 2017zzts212).

\section{Availability of data and materials}

The datasets used and/or analyzed during the current study are available from the corresponding author on reasonable request.

\section{Authors' contributions}

$\mathrm{HZ}$ and MT designed the experiments. YW and GY performed the experiments. HY and ZZ contributed to data analysis. YW wrote the manuscript. All authors read and approved the final manuscript.

\section{Ethics approval and consent to participate}

The study protocol was approved by the Ethics Committee of Xiangya Hospital of Central South University (Protocol ID: 201703358). Each bone marrow donor signed informed consent before the study.

\section{Patient consent for publication}

Not applicable.

\section{Competing interests}

The authors declare that they have no competing interests.

\section{References}

1. Loeser RF, Collins JA and Diekman BO: Ageing and the pathogenesis of osteoarthritis. Nat Rev Rheumatol 12: 412-420, 2016.

2. Makris EA, Gomoll AH, Malizos KN, Hu JC and Athanasiou KA: Repair and tissue engineering techniques for articular cartilage. Nat Rev Rheumatol 11: 21-34, 2015.

3. Roos EM and Arden NK: Strategies for the prevention of knee osteoarthritis. Nat Rev Rheumatol 12: 92-101, 2016.

4. Bhattacharjee M, Coburn J, Centola M, Murab S, Barbero A, Kaplan DL, Martin I and Ghosh S: Tissue engineering strategies to study cartilage development, degeneration and regeneration. Adv Drug Deliv Rev 84: 107-122, 2015.

5. Evans $\mathrm{CH}$ and Huard J: Gene therapy approaches to regenerating the musculoskeletal system. Nat Rev Rheumatol 11: 234-242, 2015.

6. Craft AM, Rockel JS, Nartiss Y, Kandel RA, Alman BA and Keller GM: Generation of articular chondrocytes from human pluripotent stem cells. Nat Biotechnol 33: 638-645, 2015.

7. Xia H, Liang C, Luo P, Huang J, He J, Wang Z, Cao X, Peng C and $\mathrm{Wu}$ S: Pericellular collagen I coating for enhanced homing and chondrogenic differentiation of mesenchymal stem cells in direct intra-articular injection. Stem Cell Res Ther 9: 174, 2018.

8. Grayson WL, Bunnell BA, Martin E, Frazier T, Hung BP and Gimble JM: Stromal cells and stem cells in clinical bone regeneration. Nat Rev Endocrinol 11: 140-150, 2015.

9. Visweswaran M, Pohl S, Arfuso F, Newsholme P, Dilley R, Pervaiz S and Dharmarajan A: Multi-lineage differentiation of mesenchymal stem cells-To Wnt, or not Wnt. Int J Biochem Cell Biol 68: 139-147, 2015.

10. Jiang $X$, Huang $X$, Jiang T, Zheng L, Zhao J and Zhang X: The role of Sox 9 in collagen hydrogel-mediated chondrogenic differentiation of adult mesenchymal stem cells (MSCs). Biomater Sci 6: 1556-1568, 2018.

11. Loebel C, Czekanska EM, Bruderer M, Salzmann G, Alini M and Stoddart MJ: In vitro osteogenic potential of human mesenchymal stem cells is predicted by Runx2/Sox9 ratio. Tissue Eng Part A 21: 115-123, 2015.

12. Ono N, Ono W, Nagasawa T and Kronenberg HM: A subset of chondrogenic cells provides early mesenchymal progenitors in growing bones. Nat Cell Biol 16: 1157-1167, 2014.

13. Shivdasani RA: MicroRNAs: Regulators of gene expression and cell differentiation. Blood 108: 3646-3653, 2006.

14. Treiber T, Treiber N and Meister G: Regulation of microRNA biogenesis and its crosstalk with other cellular pathways. Nat Rev Mol Cell Biol 20: 5-20, 2019.

15. Wa Q, He P, Huang S, Zuo J, Li X, Zhu J, Hong S, Lv G, Cai D, $\mathrm{Xu} \mathrm{D}$, et al: miR-30b regulates chondrogenic differentiation of mouse embryo-derived stem cells by targeting SOX9. Exp Ther Med 14: 6131-6137, 2017.

16. Anderson BA and McAlinden A: miR-483 targets SMAD4 to suppress chondrogenic differentiation of human mesenchymal stem cells. J Orthop Res 35: 2369-2377, 2017.

17. Lee S, Yoon DS, Paik S, Lee KM, Jang Y and Lee JW: microRNA-495 inhibits chondrogenic differentiation in human mesenchymal stem cells by targeting Sox9. Stem Cells Dev 23: 1798-1808, 2014.

18. Moutinho C and Esteller M: MicroRNAs and epigenetics. Adv Cancer Res 135: 189-220, 2017.

19. van Meurs JB, Boer CG, Lopez-Delgado L and Riancho JA: Role of epigenomics in bone and cartilage disease. J Bone Miner Res 34: 215-230, 2019.

20. Chang T, Xie J, Li H, Li D, Liu P and Hu Y: MicroRNA-30a promotes extracellular matrix degradation in articular cartilage via downregulation of Sox9. Cell Prolif 49: 207-218, 2016.

21. Almeida MI, Silva AM, Vasconcelos DM, Almeida CR, Caires H, Pinto MT, Calin GA, Santos SG and Barbosa MA: miR-195 in human primary mesenchymal stromal/stem cells regulates proliferation, osteogenesis and paracrine effect on angiogenesis. Oncotarget 7: 7-22, 2016.

22. Liu S, Dong H, Dai H, Liu D and Wang Z: MicroRNA-216b regulated proliferation and invasion of non-small cell lung cancer by targeting SOX9. Oncol Lett 15: 10077-10083, 2018.

23. Liu XJ, Bai XG, Teng YL, Song L, Lu N and Yang RQ: miRNA-15a-5p regulates VEGFA in endometrial mesenchymal stem cells and contributes to the pathogenesis of endometriosis. Eur Rev Med Pharmacol Sci 20: 3319-3326, 2016. 
24. Wang YJ, Zhang HQ, Han HL, Zou YY, Gao QL and Yang GT: Taxifolin enhances osteogenic differentiation of human bone marrow mesenchymal stem cells partially via NF- $\mathrm{KB}$ pathway. Biochem Biophys Res Commun 490: 36-43, 2017.

25. Livak KJ and Schmittgen TD: Analysis of relative gene expression data using real-time quantitative PCR and the 2(-Delta Delta C(T)) method. Methods 25: 402-408, 2001.

26. Uder C, Bruckner S, Winkler S, Tautenhahn HM and Christ B: Mammalian MSC from selected species: Features and applications. Cytometry A 93: 32-49, 2018.

27. Zhao C, Jiang W, Zhou N, Liao J, Yang M, Hu N, Liang X, Xu W, Chen H, Liu W, et al: Sox9 augments BMP2-induced chondrogenic differentiation by downregulating Smad7 in mesenchymal stem cells (MSCs). Genes Dis 4: 229-239, 2017.

28. Liao J, Hu N, Zhou N, Lin L, Zhao C, Yi S, Fan T, Bao W, Liang X, Chen H, et al: Sox9 potentiates BMP2-induced chondrogenic differentiation and inhibits BMP2-induced osteogenic differentiation. PLoS One 9: e89025, 2014.

29. Almalki SG and Agrawal DK: Key transcription factors in the differentiation of mesenchymal stem cells. Differentiation 92 $41-51,2016$

30. Chang $M$, Lin $H$, Fu H, Wang B, Han $G$ and Fan $M$ : MicroRNA-195-5p regulates osteogenic differentiation of periodontal ligament cells under mechanical loading. J Cell Physiol 232: 3762-3774, 2017.

31. Eguchi T, Watanabe K, Hara ES, Ono M, Kuboki T and Calderwood SK: OstemiR: A novel panel of microRNA biomarkers in osteoblastic and osteocytic differentiation from mesencymal stem cells. PLoS One 8: e58796, 2013.

32. Luo Y, Sinkeviciute D, He Y, Karsdal M, Henrotin Y, Mobasheri A, Önnerfjord P and Bay-Jensen A: The minor collagens in articular cartilage. Protein Cell 8: 560-572, 2017.

33. Duan P and Bonewald LF: The role of the wnt $/ \beta$-catenin signaling pathway in formation and maintenance of bone and teeth. Int J Biochem Cell Biol 77: 23-29, 2016.

34. Deng A, Zhang H, Hu M, Liu S, Wang Y, Gao Q and Guo C: The inhibitory roles of Ihh downregulation on chondrocyte growth and differentiation. Exp Ther Med 15: 789-794, 2018.

35. Deng ZH, Li YS, Gao X, Lei GH and Huard J: Bone morphogenetic proteins for articular cartilage regeneration. Osteoarthritis Cartilage 26: 1153-1161, 2018

36. Viswanathan C, Kulkarni R, Bopardikar A and Ramdasi S: Significance of CD34 negative hematopoietic stem cells and CD34 positive mesenchymal stem cells-A valuable dimension to the current understanding. Curr Stem Cell Res Ther 12: 476-483, 2017.

37. Szade K, Zuba-Surma E, Rutkowski AJ, Jozkowicz A and Dulak J: CD45-CD14+CD34+ murine bone marrow low-adherent mesenchymal primitive cells preserve multilineage differentiation potential in long-term in vitro culture. Mol Cells 31: 497-507, 2011.

38. Baptista LS, do Amaral RJ, Carias RB, Aniceto M, Claudio-da-Silva $\mathrm{C}$ and Borojevic R: An alternative method for the isolation of mesenchymal stromal cells derived from lipoaspirate samples. Cytotherapy 11: 706-715, 2009.

39. Healy C, Uwanogho D and Sharpe PT: Regulation and role of Sox9 in cartilage formation. Dev Dyn 215: 69-78, 1999.
40. Somoza RA, Welter JF, Correa D and Caplan AI: Chondrogenic differentiation of mesenchymal stem cells: Challenges and unfulfilled expectations. Tissue Eng Part B Rev 20: 596-608, 2014.

41. Barry F and Murphy M: Mesenchymal stem cells in joint disease and repair. Nat Rev Rheumatol 9: 584-594, 2013.

42. Jo A, Denduluri S, Zhang B, Wang Z, Yin L, Yan Z, Kang R, Shi LL, Mok J, Lee MJ and Haydon RC: The versatile functions of Sox 9 in development, stem cells, and human diseases. Genes Dis 1: 149-161, 2014

43. Shim J and Nam JW: The expression and functional roles of microRNAs in stem cell differentiation. BMB Rep 49: 3-10, 2016.

44. Martin EC, Qureshi AT, Llamas CB, Burow ME, King AG, Lee OC, Dasa V, Freitas MA, Forsberg JA, Elster EA, et al: Mirna biogenesis pathway is differentially regulated during adipose derived stromal/stem cell differentiation. Adipocyte 7: 96-105, 2018

45. Zeng ZL, Lin XL, Tan LL, Liu YM, Qu K and Wang Z: MicroRNAs: Important regulators of induced pluripotent stem cell generation and differentiation. Stem Cell Rev 14: 71-81, 2018.

46. Ran X, Xiao CH, Xiang GM and Ran XZ: Regulation of embryonic stem cell self-renewal and differentiation by MicroRNAs. Cell Reprogramm 19: 150-158, 2017.

47. Papaioannou G, Mirzamohammadi F, Lisse TS, Nishimori S, Wein MN and Kobayashi T: MicroRNA-140 provides robustness to the regulation of hypertrophic chondrocyte differentiation by the PTHrP-HDAC4 pathway. J Bone Miner Res 30: 1044-1052, 2015.

48. Zhang Y, Huang X and Yuan Y: MicroRNA-410 promotes chondrogenic differentiation of human bone marrow mesenchymal stem cells through down-regulating Wnt3a. Am J Transl Res 9: 136-145, 2017.

49. Suchorska WM, Augustyniak E, Richter M and Trzeciak T: Gene expression profile in human induced pluripotent stem cells: Chondrogenic differentiation in vitro, part A. Mol Med Rep 15: 2387-2401, 2017.

50. Oh CD, Yasuda H, Zhao W, Henry SP, Zhang Z, Xue M, de Crombrugghe B and Chen D: SOX9 directly regulates $\mathrm{CTGF} / \mathrm{CCN} 2$ transcription in growth plate chondrocytes and in nucleus pulposus cells of intervertebral disc. Sci Rep 6: 29916, 2016.

51. Maciejak A, Kostarska-Srokosz E, Gierlak W, Dluzniewski M, Kuch M, Marchel M, Opolski G, Kiliszek M, Matlak K, Dobrzycki S, et al: Circulating miR-30a-5p as a prognostic biomarker of left ventricular dysfunction after acute myocardial infarction. Sci Rep 8: 9883, 2018

52. Zhang C, Ma X, Du J, Yao Z, Shi T, Ai Q, Chen X, Zhang Z, Zhang $X$ and Yao X: MicroRNA-30a as a prognostic factor in urothelial carcinoma of bladder inhibits cellular malignancy by antagonising Notch1. BJU Int 118: 578-589, 2016.

53. Perugorria MJ, Olaizola P, Labiano I, Esparza-Baquer A, Marzioni M, Marin JJG, Bujanda L and Banales JM: Wnt- $\beta$-catenin signalling in liver development, health and disease. Nat Rev Gastroenterol Hepatol 16: 121-136, 2019.

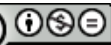

This work is licensed under a Creative Commons Attribution-NonCommercial-NoDerivatives 4.0 International (CC BY-NC-ND 4.0) License. 